\title{
A single-center comparative study of lung ultrasound versus chest computed tomography during the COVID-19 era
}

\author{
Kobalava Zhanna Davidovna, ${ }^{1}$ Safarova Ayten Fuad, ${ }^{1,2}$ Flora Elisa Cabello Montoya, ${ }^{1}$ Maria Vasilevna Vatsik- \\ Gorodetskaya, ${ }^{2}$ Karaulova Yulia Leonidovna, ${ }^{1}$ Zorya Olga Tairovna, ${ }^{1}$ Arutina Olga Valeryevna, ${ }^{1}$ Rajesh Rajan, ${ }^{1,3}$ \\ Mohammed Al Jarallah, ${ }^{3}$ Peter A. Brady, ${ }^{4}$ Ibrahim Al-Zakwani ${ }^{5}$ \\ ${ }^{1}$ Department of Internal Medicine with the subspecialty of cardiology and functional diagnostics named after V.S. Moiseev, \\ Institute of Medicine, Peoples' Friendship University of Russia (RUDN University), Moscow, Russia \\ ${ }^{2}$ Clinical Hospital named after V.V. Vinogradov Moscow, Moscow, Russia \\ ${ }^{3}$ Department of Cardiology, Sabah Al Ahmed Cardiac Centre, Kuwait City, Kuwait \\ ${ }^{4}$ Department of Cardiology, Illinois Masonic Medical Center, Chicago IL, USA \\ ${ }^{5}$ Department of Pharmacology and Clinical Pharmacy, College of Medicine and Health Sciences, Sultan Qaboos University, \\ Muscat, Oman
}

Background: Lung ultrasound (LUS) is a bedside imaging tool that has proven useful in identifying and assessing the severity of pulmonary pathology. The aim of this study was to determine LUS patterns, their clinical significance, and how they compare to CT findings in hospitalized patients with coronavirus infection.

Methods: This observational study included 62 patients ( 33 men, age $59.3 \pm 15.9$ years), hospitalized with pneumonia due to COVID-19, who underwent chest CT and bedside LUS on the day of admission. The CT images were analyzed by chest radiographers who calculated a CT visual score based on the expansion and distribution of ground-glass opacities and consolidations. The LUS score was calculated according to the presence, distribution, and severity of anomalies.

Results: All patients had CT findings suggestive of bilateral COVID-19 pneumonia, with an average visual scoring of $8.1 \pm 2.9 \%$. LUS identified 4 different abnormalities, with bilateral distribution (mean LUS score: $26.4 \pm 6.7$ ), focal areas of non-confluent B lines, diffuse confluent B lines, small sub-pleural micro consolidations with pleural line irregularities, and large parenchymal consolidations with air bronchograms. LUS score was significantly correlated with CT visual scoring (rho $=0.70 ; \mathrm{p}<0.001)$. Correlation analysis of the CT and LUS severity scores showed good interclass correlation $(\mathrm{ICC})$ (ICC $=0.71 ; 95 \%$ confidence interval $(\mathrm{CI}): 0.52-0.83 ; \mathrm{p}<0.001)$. Logistic regression was used to determine the cut-off value of $\geq 27$ (area under the curve: $0.97 ; 95 \%$ CI: $90-99$; sensitivity $88.5 \%$ and specificity $97 \%$ ) of the LUS severity score that represented severe and critical pulmonary involvement on chest CT (CT: 3-4).

Conclusion: When combined with clinical data, LUS can provide a potent diagnostic aid in patients with suspected COVID-19 pneumonia, reflecting CT findings.

Key words: COVID-19; lung ultrasonography; computed tomography; consolidation; B-lines.

Correspondence: Dr. Rajesh Rajan, Department of Cardiology, Sabah Al Ahmed Cardiac Centre, 13001 Kuwait City, Kuwait. Tel.+965.6587332. E-mail: cardiology08@gmail.com

Contributions: KZD, SAF, FECM, MVV, KYL, participated in data analysis and manuscript preparation; ZOT, participated in the data analysis; IAZ, did the statistical analysis as well as manuscript review. All authors had access to data and take responsibility for the integrity of data and the accuracy of data analysis. All authors have read and approved the manuscript.

Conflict of interest: The authors declare no conflicts of interest.

Funding: The study was funded by RFBR project number 19-313-90058.

Ethics approval and consent to participate: The study was approved by the local Ethics Committee number 3 (Ethics Approval Letter number N3; dated: 09.07.2020).

Data availability: The data are available upon request. Contact author is Dr. Safarova Ayten Fuad, associate professor at the Department of Internal Diseases with Courses of Cardiology and Functional Diagnostics, Peoples' Friendship University of Russia (RUDN University). Postal address: 61, Vavilova street, Moscow, Russia, 117292. E-mail: aytensaf@mail.ru 


\section{Introduction}

Lung ultrasound (LUS) is a readily available bedside technique to diagnose and monitor the presence and severity of lung pathology and may be of value in patients with COVID-19 infection $[1,2]$. Computed tomography (CT) of the chest is the gold standard for diagnosing pulmonary involvement in patients who are admitted to the hospital with fever and respiratory symptoms. The high transmissibility of SARS-CoV-2 and the high risk of complications during transport of unstable patients with hypoxia or hemodynamic instability may limit our ability to perform CT scans on patients with likely or verified COVID-19 infection. The major advantages of LUS are: low cost, ready availability, and the possibility of performing it at bedside. LUS can also be performed both in patients breathing independently and those being mechanically ventilated. LUS is also safe if repeated imaging is necessary since there is no ionizing radiation.

Lung involvement in COVID-19 pneumonia is generally seen in the subpleural areas, which can be easily visualized with ultrasound. Several studies have shown that in SARS-CoV-2 pneumonia, ultrasound findings may include multifocal B-lines, bilateral subpleural thickening and pleural thickening, which correspond with changes seen on CT imaging [1,3]. Unfortunately, there is only a handful of studies that explore the correlation between LUS and CT findings. The aim of the current study was to determine the performance of LUS in diagnosing severe SARS-CoV-2 pneumonia in comparison with chest $\mathrm{CT}$.

\section{Methods}

This prospective observational study was performed at City Clinical Hospital named after V.V. Vinogradov (Moscow, Russia) with subjects from both the medical floors and the intensive care unit (ICU) with suspected COVID-19 infection. The study included individuals aged 18 and older with either verified or likely coronavirus related pneumonia. Verified patients were those in whom SARS-CoV-2 was positive on PCR and also had characteristic pulmonary involvement. Patients considered likely to have the infection were those with a negative PCR but characteristic pulmonary findings. The study was approved by the local Ethics Committee number 3 (Ethics Approval Letter number N3; dated: 09.07.2020). Exclusion criteria included patients with inadequate lung visualization on ultrasound due to either obesity or emphysema, decompensated congestive heart failure, prior interstitial lung disease, tuberculosis, lung neoplasm, pulmonary embolism, extremely ill patients with very poor prognosis.

Disease severity was classified according to criteria proposed by Interim Guidance on COVID-19 developed by World Health Organization (WHO), which classified patients into four groups of severity: mild, moderate, severe, and critical [4]. The diagnosis of SARS-CoV-2 infection was made by identification of viral RNA via PCR from upper respiratory tract mucosal samples (combined naso-oropharyngeal swab). Non-contrast chest CT was performed using the Aquilion CXL with the patient in supine position. Images were retrieved and reconstructed as axial images with the following parameters: slice thickness $1.0 \mathrm{~mm}$, interval $0.7 \mathrm{~mm}, 120 \mathrm{kV}$. The following findings that were considered characteristic of SARS-CoV-2 pneumonia include: bilateral ground glass opacities, reticular changes overlying ground glass opacities, peripheral consolidation and perilobular thickening [5] (Figure 1).

The severity of lung involvement was classified according to the quantity and quality of involvement of lung parenchyma: CT-1

(ground glass opacities in $\leq 25 \%$ of lung parenchyma); CT-2 (ground glass opacities in $26-50 \%$ of lung parenchyma); CT-3 (ground glass opacities and consolidations in $51-75 \%$ of lung parenchyma); CT-4 (ground glass opacities and consolidations with reticular changes in $\geq 76 \%$ of lung parenchyma). The lesions extent within each lung lobe were evaluated by scoring from 0 to 4 (Table 1). Summation of scores from all 5 lobes provided the total CT severity score ranging from 0 to 20 [6-9].

LUS was performed using the portable VIVID iq (GE) machine with 3.5-5 MHz convex probes. Special covers were used for the probes and the machine during the procedure. We followed an established ultrasound algorithm to minimize the risks of infection transmission and the duration of the procedure. During the ultrasound examinations all images were recorded and analyzed off-line. The person performing the ultrasound was blinded to the CT scan results. LUS was performed with patients in a seated or supine position depending on the clinical status. A modified 16lung zones scanning protocol was used. A detailed description of the zones is described in Table 2 and in Figure 2. Each chest area includes 2-3 intercostal spaces. The lesions extent was evaluated semi-quantitatively using the modified scale [10]. Each of the 16 examined areas was evaluated by scoring 0 to 3 (Table 3 ). Summation of the scores determined the severity of lung involvement: a score of 0 represented the normal lung, whereas the score of 48 represented very severe damage. The results were registered in the protocol (Figure 3).

\section{Statistical analysis}

Numerical variables with normal distribution were presented as mean and standard deviation. Variables that were not normally distributed were reported as median and interquartile range. Categorical variables were presented as frequencies and percent-

Table 1. Scale for assessing the severity of lung damage in COVID -19 according to the computer tomography of the chest organs.

\begin{tabular}{lc} 
Points & Degree of lung damage, $\%$ \\
0 & 0 \\
1 & $1-25 \%$ \\
\hline 2 & $26-50 \%$ \\
3 & $51-75 \%$ \\
\hline 4 & $>76 \%$ \\
\hline
\end{tabular}

a

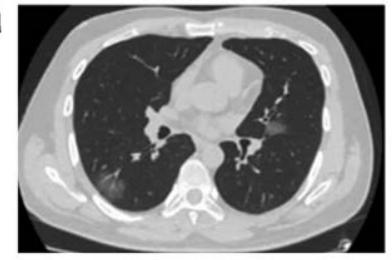

b
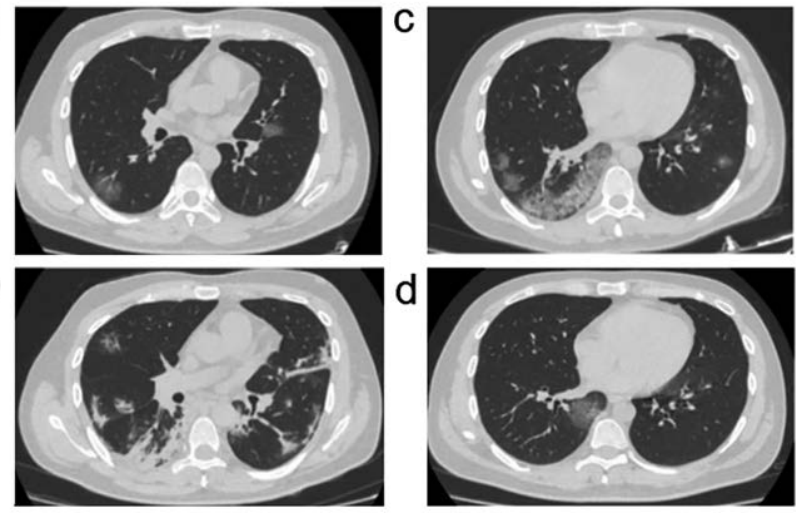

Figure 1. High-resolution computed scan: a,d) ground-glass opacities; b) perilobular thickening, reticular changes overlying ground glass opacities (crazy-paving pattern); c) consolidations. 
ages. Comparisons between groups were made by the non-parametric Mann-Whitney $U$ test for numerical variables and by Pearson's chi-squared test for categorical variables. The correlation of the LUS score with the CT score and oxygen saturation in room air was calculated with Spearman correlation index. The intraclass correlation coefficient (ICC) was used to assess similarity of LUS and CT scores. An ICC index $<0.4,0.4-0.75$ and $>0.75$ represented low, moderate and high similarity between values, respectively. Receiver operating characteristic (ROC) analysis with area under the ROC-curve (AUC) measurement was used to assess diagnostic efficacy of the calculated scores. The most appropriate cut-off value with the highest sum of sensitivity and specificity was determined. A $\mathrm{p}<0.05$ was considered statistically significant. Statistical analysis was performed using Statistica Version 20.0 and MedCalc Software's VAT Version 19.0. required oxygen supplementation including high-flow nasal oxygen $(3.2 \%)$, non-invasive ventilation $(4.8 \%)$ and $6.5 \%$ of patients required mechanical ventilation. All patients had bilateral lung involvement on chest CT. The most common types of lesions included ground glass opacities and consolidation; the majority of patients had lung involvement that corresponded to severity stages CT-2 and CT-3 (Table 6). The most common LUS patterns included patches of B-lines, pleural thickening with multiple sub-pleural lesions and an irregular pleural line (Figure 4). Pleural effusion was identified in $3(4.8 \%)$ patients. Mean CT severity score was $7.9 \pm 3.2$ and mean LUS severity score was $26.4 \pm 6.7$. The comparison of lung involvement severity according to the chest CT and the total CT and LUS severity scores is presented in Table 7 and
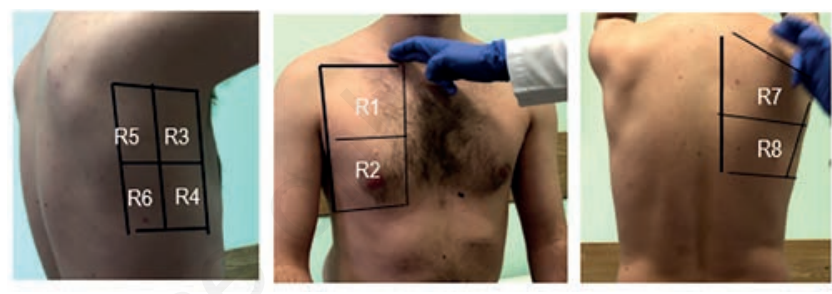

Figure 2. Proposal for lung ultrasound scanning scheme in COVID-19 patients. Each chest area includes 2-3 intercostal spaces. The lesions extent was evaluated semi-quantitatively using the modified scale [10]. Each of the 16 examined areas was evaluated by scoring 0 to 3 (Table 3 ).

Table 2. Anatomical landmarks and zones when using a 16-zone lung ultrasound protocol.

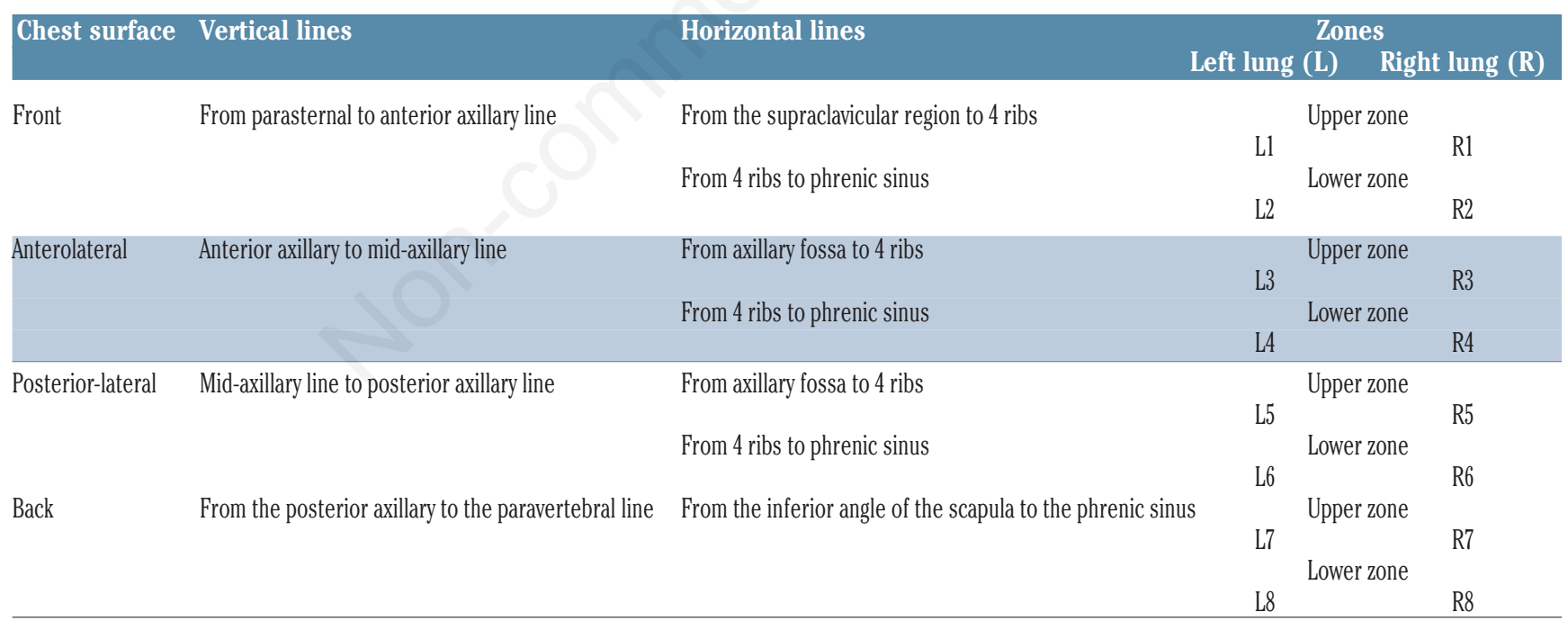

Table 3. US scale for assessing the severity of lung injury in COVID-19.

\begin{tabular}{lll} 
Points & Loss rate airiness of the lungs & Ultrasound pattern \\
0 & Norm & Horizontal A-lines or B-lines $<3$ in the scan area \\
1 & Moderate degree & Multiple B-lines (B-lines $\geq 3$ or confluent B-lines $\leq 50$ in the scan area) without subpleural lesions \\
\hline $1 s$ & Moderate degree & Multiple B lines (confluent B lines $\leq 50 \%$ in the scanned area) with subpleural lesions \\
2 & Severe degree & Multiple B-lines (confluent B-lines $>50 \%$ in the scan area) without subpleural lesions \\
$2 \mathrm{~s}$ & Severe degree & Multiple B lines (confluent B lines $>50 \%$ in the scan area) with subpleural lesions \\
3 & Complete loss of airiness & Consolidation (aerobronchogram $+/-$ )
\end{tabular}


Figure 5 .

Correlation analysis of the CT and LUS severity scores showed good ICC $(0.71 ; 95 \%$ confidence interval (CI): $0.52-0.83$; $\mathrm{p}<0.001)$. These results signify that both LUS and CT severity scores are similarly reliable for evaluating lung involvement in patients with COVID-19. LUS severity scores also strongly correlated with CT severity classes ( $r h o=0.70 ; p<0.001)$ (Figure 6). LUS severity scores were stratified into two groups: above and below the median $(\geq 26$ and $<26)$. Patients in the first group $(\geq 26)$ had more lung involvement on the CT scans compared with the patients in the second group $(<26)(\mathrm{rho}=0.62 ; \mathrm{p}<0.001)$ (Figure 7).

Total LUS severity scores correlated more strongly with disease severity classification $(\mathrm{rho}=0.502 ; \mathrm{p}<0.000), \mathrm{C}$-reactive protein $(\mathrm{rho}=0.442 ; \mathrm{p}<0.001)$ and d-dimer levels $(\mathrm{rho}=0.28 ; \mathrm{p}=0.02)$ than total CT severity score $(\mathrm{rho}=0.39 ; \mathrm{p}=0.001$ : $\mathrm{rho}=0.38 ; \mathrm{p}=0.002$ : rho $=0.28 ; \mathrm{p}=0.03$, respectively). We also found negative correlation between the total LUS severity scores and blood oxygen saturation at admission that was statistically significant ( $r h o=-0.25 ; \mathrm{p}=0.04$ ). Meanwhile, the correlation between total CT scores and oxygen saturation was not statistically significant $(\mathrm{rho}=-0.21 ; \mathrm{p}=0.09)$. Logistic regression was used to determine the cut-off value $\geq 27$ (AUC, 0.967; 95\%CI: $90 \%-99 \%$; sensitivity $89 \%$; specificity $97 \%$ ) of the LUS severity score that represented severe and critical pulmonary involvement on chest CT (CT 3-4) (Figure 8).

Table 4. Clinical and demographic characteristics of patients.

\begin{tabular}{|c|c|}
\hline Characteristic & Value \\
\hline Number of patients & 62 \\
\hline Gender (m/f), n (\%) & $33(53 \%) / 29(47 \%)$ \\
\hline Age, mean \pm SD, years & $59 \pm 16$ \\
\hline $\mathrm{BMI}$, mean $\pm \mathrm{SD}, \mathrm{kg} / \mathrm{m}^{2}$ & $30.1 \pm 6.0$ \\
\hline Systolic blood pressure, median (IQR), mmHg & $133 \pm 20$ \\
\hline Diastolic blood pressure, median (IQR), mmHg & $80 \pm 12$ \\
\hline Heart rate, mean \pm SD, bpm & $91 \pm 13$ \\
\hline Respiratory rate, mean \pm SD, rpm & $21 \pm 2.0$ \\
\hline $\mathrm{SO}_{2}$, mean $\pm \mathrm{SD}, \%$ & $93 \pm 3.2$ \\
\hline Temperature, mean $\pm \mathrm{SD},{ }^{\circ} \mathrm{C}$ & $38 \pm 0.8$ \\
\hline $\begin{array}{l}\text { Disease severity } \\
\text { Moderate, n (\%) } \\
\text { Severe, n (\%) }\end{array}$ & $\begin{array}{l}46(74 \%) \\
16(26 \%)\end{array}$ \\
\hline $\begin{array}{l}\text { Comorbidities }(\mathrm{n}=44) \\
\text { Hypertension, } \mathrm{n}(\%) \\
\text { Chronic heart failure, } \mathrm{n}(\%) \\
\text { Diabetes mellitus 2, } \mathrm{n}(\%) \\
\text { Ischemic cardiomyopathy/CAD, n (\%) } \\
\text { Atrial fibrillation, n (\%) } \\
\text { COPD, n (\%) } \\
\text { Stroke, n (\%) } \\
\text { Malignancy, n (\%) }\end{array}$ & $\begin{array}{l}41(93 \%) \\
21(48 \%) \\
13(30 \%) \\
10(23 \%) \\
6(14 \%) \\
6(14 \%) \\
4(9.1 \%) \\
4(9.1 \%)\end{array}$ \\
\hline $\begin{array}{l}\text { Symptoms } \\
\text { Dyspnea, n (\%) } \\
\text { Fever, n (\%) } \\
\text { Cough, n (\%) } \\
\text { Anosmia/ageusia, n (\%) } \\
\text { Diarrhea, n (\%) }\end{array}$ & $\begin{array}{c}62(100) \\
60(96,8) \\
54(87,1) \\
7(11,3) \\
3(2,4)\end{array}$ \\
\hline
\end{tabular}

$\mathrm{SD}$, standard deviation; $\mathrm{IQR}$, interquartile range; $b p m$, beats per minute; rpm, respiratory rate per minute; $\mathrm{SO}_{2}$, oxygen saturation; $\mathrm{CAD}$, coronary artery disease; $\mathrm{COPD}$, chronic obstructive airways disease.
Table 5. Laboratory characteristics of patients.

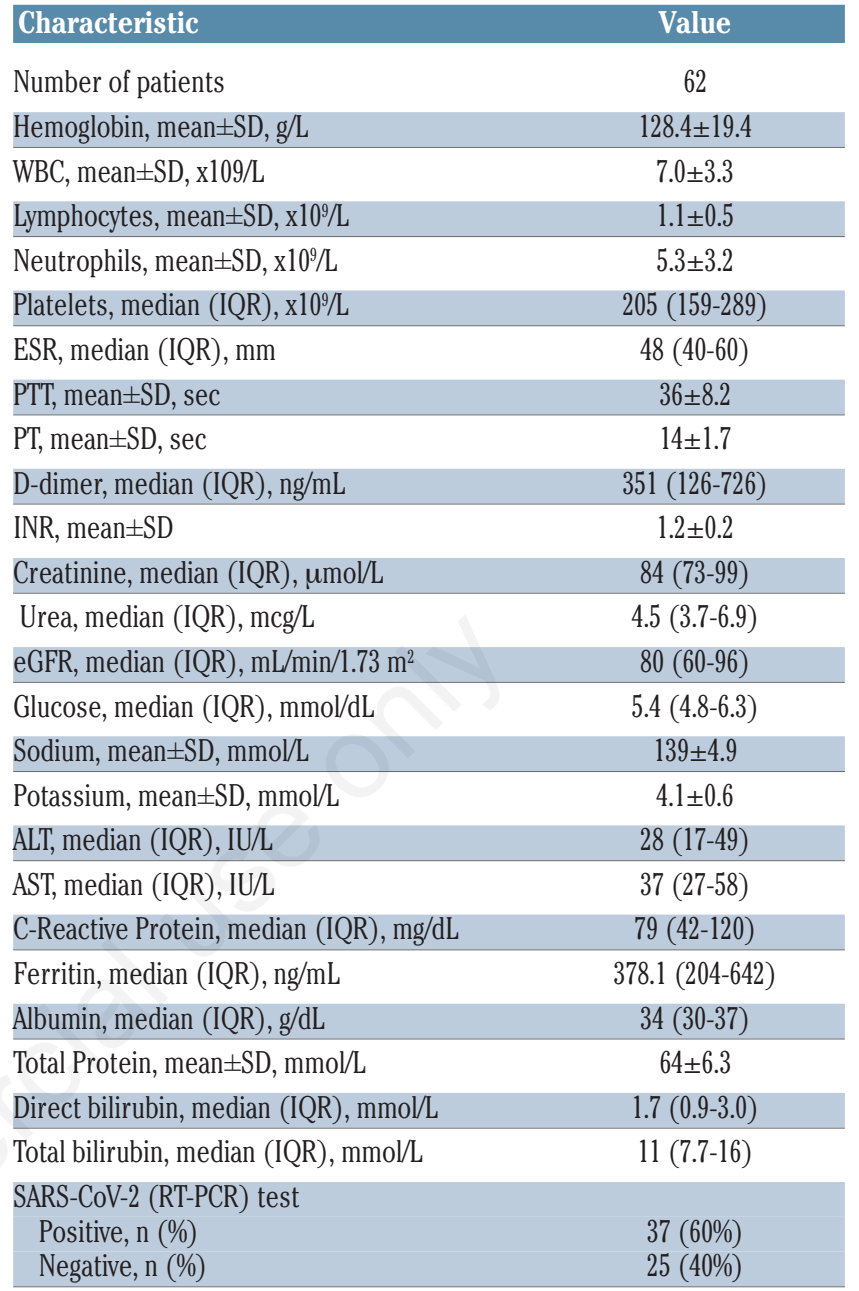

SD, standard deviation; WBC, white blood cell count; IQR, interquartile range; ESR, erythrocyte sedimentation rate; PTT, partial thromboplastin time; PT, prothrombin time, INR, international normalized ratio; eGFR, estimated glomerular filtration rate; ALT, alanine aminotransferase; ASR, aspartate aminotransferase.

Table 6. The degree of lung lesions by CT and ultrasound of the lungs. Nature and extent of the lesion on CT

\begin{tabular}{ll} 
Proportion of persons with bilateral lesions, $\mathrm{n}(\%)$ & $62(100)$ \\
CT- $1,<25 \%, \mathrm{n}(\%)$ & $10(16 \%)$ \\
\hline CT- $2,26-50 \%, \mathrm{n}(\%)$ & $26(42 \%)$ \\
CT -3, 51-75\%, n (\%) & $24(39 \%)$ \\
\hline CT- $4,>75 \%, \mathrm{n}(\%)$ & $2(3.2 \%)$ \\
Points total & $8.1 \pm 2.9$ \\
\hline Ultrasound of the lungs, patterns & $62(100 \%)$ \\
Bilateral defeat, n (\%) & $60(97 \%)$ \\
Subpleural consolidations, n (\%) & $48(77 \%)$ \\
Consolidation, n (\%) & $26.4 \pm 6.7$ \\
\hline
\end{tabular}




\section{Right lung}

Front surface

Side surface

Front

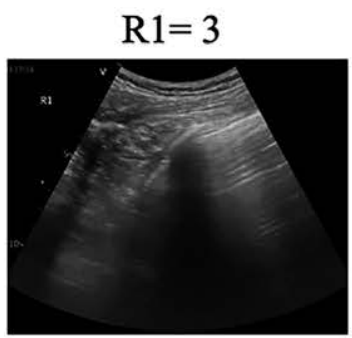

$\mathrm{R} 2=3$

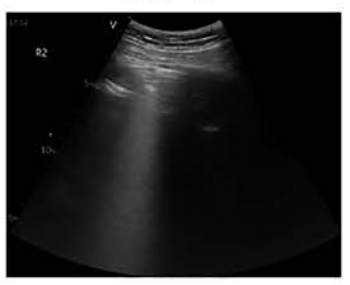

\section{Left lung}

Front surface

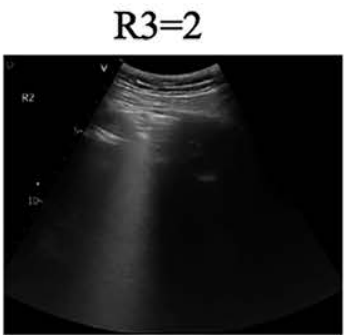

$\mathrm{R} 4=2 \mathrm{~s}$

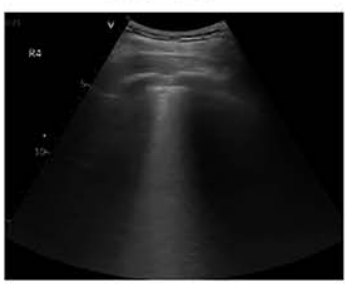

Side surface
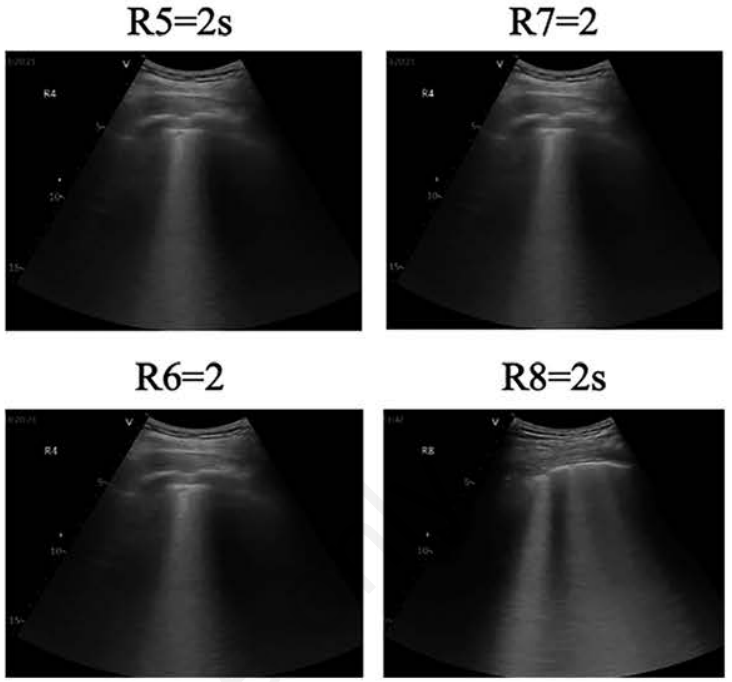

Back surface

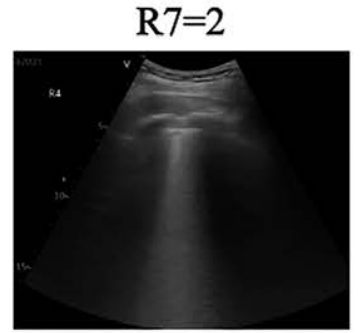

Front

Back

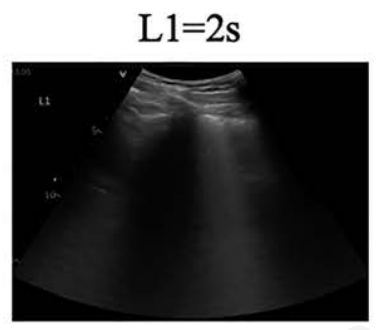

L3 $=2 \mathrm{~s}$

L5 $=2 \mathrm{~s}$

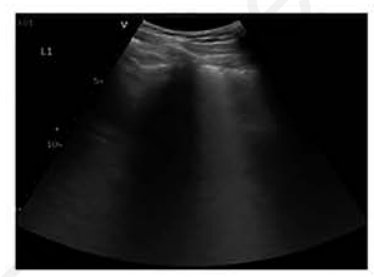

$\mathrm{L} 4=2 \mathrm{~s}$

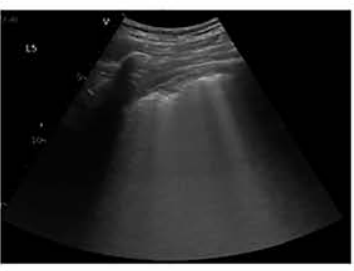

$\mathrm{L} 6=2 \mathrm{~s}$
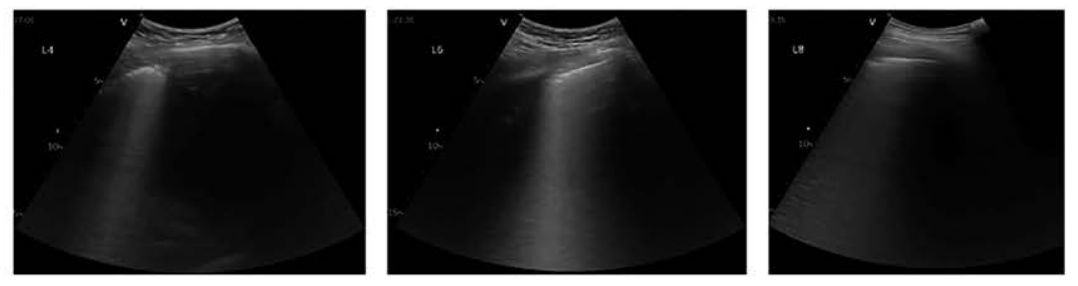

Figure 3. Protocol of ultrasound examination of patients with COVID-19 using a 16-zone protocol. Score=34.

Table 7. Comparison of the severity of lung lesions on CT thorax (\%) with the sum of scores for assessing the severity of the lesion on CT and ultrasound of the lungs.

\begin{tabular}{lcc} 
Lung lesion on chest CT & CT thorax (points total) & Ultrasound of the lungs (points total) \\
$1-25 \%$ & $4.2 \pm 1.7^{* *}$ & $17.0 \pm 3.9^{* *}$ \\
$26-50 \%$ & $6.6 \pm 1.4^{* *}$ & $23.9 \pm 2.8^{* *}$ \\
$\geq 51 \%$ & $10.7 \pm 2.8^{* *}$ & $29.9 \pm 6.3^{*}$ \\
\hline
\end{tabular}




\section{Discussion}

In our study out of the 62 patients who participated, only $60 \%$ had positive SARS-CoV-2 PCR test at admission. Other patients had seropositive rates of IgM antibodies to SARS-CoV-2. The use of standardized scanning protocol evaluates general lung aeration via the summation of scores given to each lung area. Higher LUS scores were associated with more severe lung involvement. At the same time, patients with COVID-19 can present with various LUS patterns that can also affect management approaches. The cut-off value of 27 that was determined in our study by ROC-analysis was highly sensitive (89\%) and specific (97\%) and corresponds to CT 3-4 stages of lung involvement. Importantly, LUS scores show

a
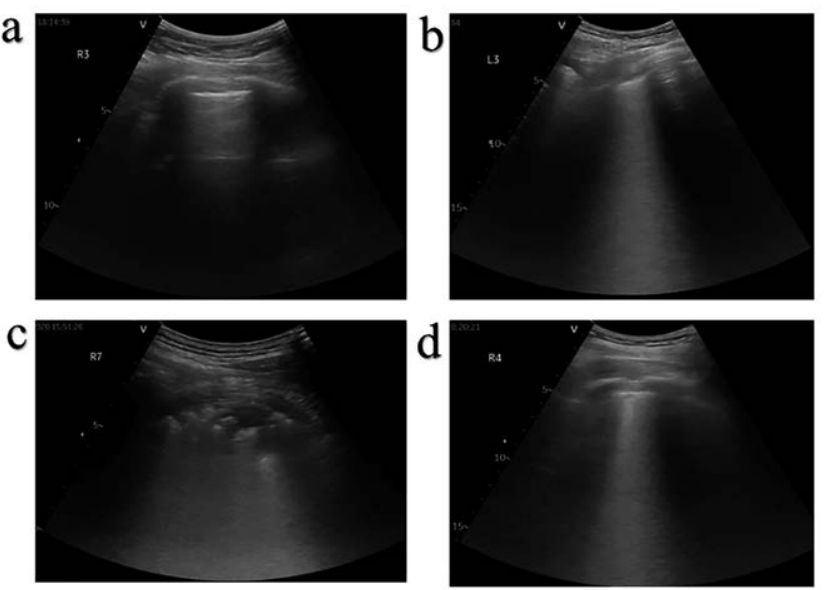

Figure 4. Appearance of COVID-19-related alveolar-interstitial pneumonia at bedside lung ultrasound. a) Spared area showing A lines corresponding to a region of normally ventilated lung parenchyma without alveolar-interstitial involvement. b) Confluent B lines with "white lung" pattern and spared areas of normal lung parenchyma showing $A$ lines. c) Overt subpleural consolidation with air bronchograms. d) Subpleural microconsolidations with indentation of pleural line, associated with a nonconfluent focal B-line pattern.

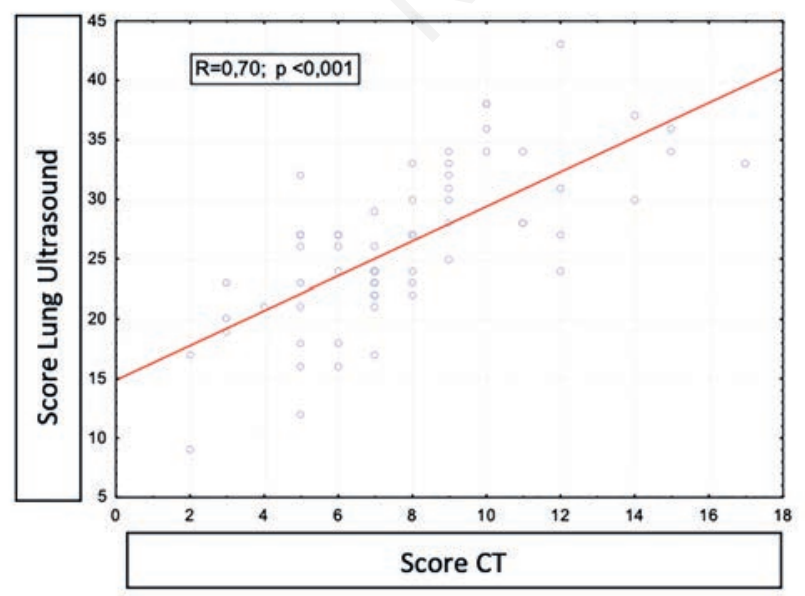

Figure 5. Spearman correlation between lung ultrasound (LUS) score and CT score.

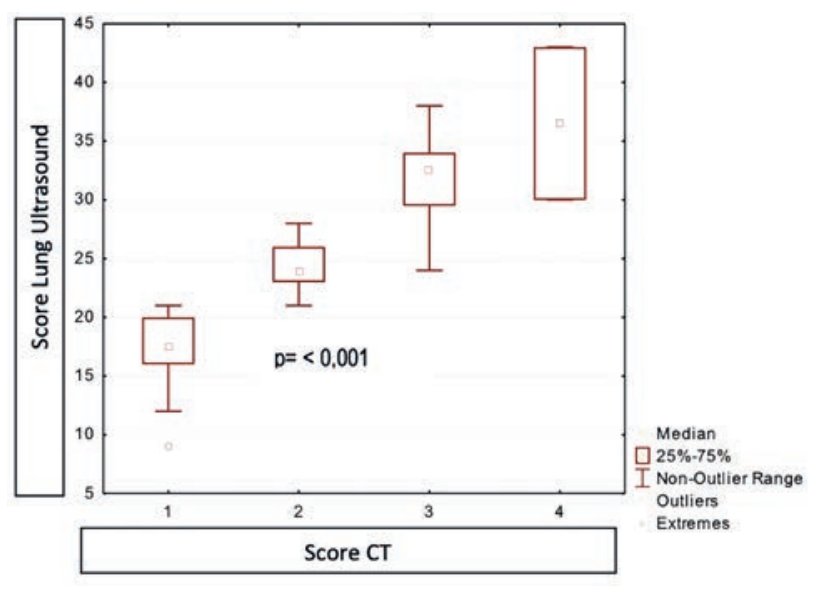

Figure 6. Median B-lines (ultrasound of the lungs), depending on the severity of lung lesions on CT. The CT score was significantly different $(p=0.016)$ between patients with LUS score below and above the median value.

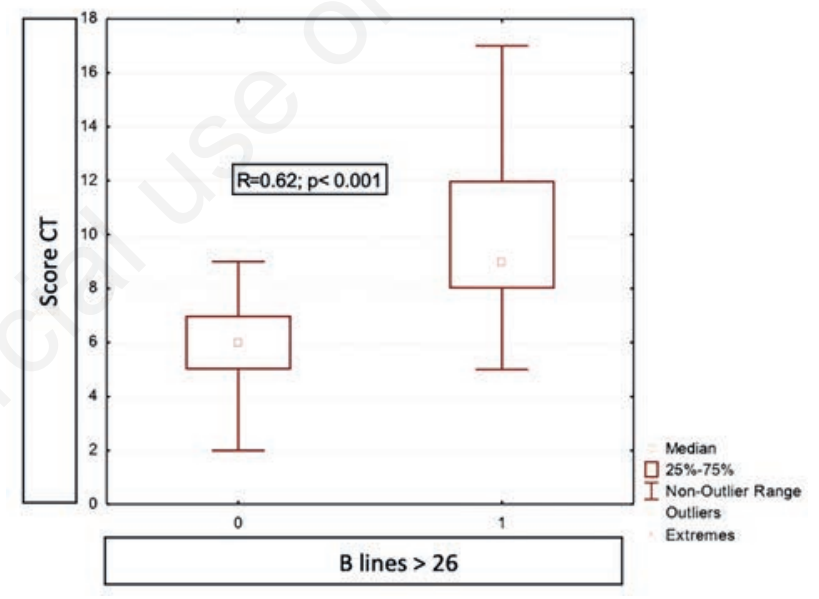

Figure 7. The CT score was significantly different $(\mathrm{p}<\mathbf{0 . 0 0 1})$ between patients with LUS score below and above the median value.

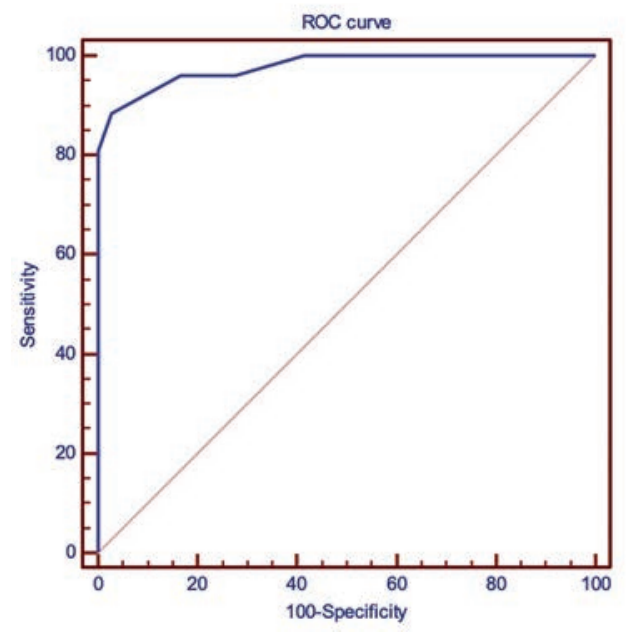

Figure 8. ROC-curve of the sensitivity and specificity of ultrasound of the lungs in assessing the severity of lung damage more than $50 \%$ in COVID-19 on a 16-zonal scale (AUC 0.98, threshold value 227 ). 
direct and significant correlation with disease severity classification, concentrations of C-reactive protein and d-dimer.

The COVID-19 infection is associated with high levels of transmission and increased mortality. Early diagnosis is essential to identify and rapidly isolate affected individuals to prevent further transmission and prevent disease progression. COVID-19 diagnosis is confirmed by PCR testing that detects viral RNA. However, this method has low sensitivity and in some cases the diagnosis is made based on clinical presentation alone in the absence of positive PCR test results. Out of all the 62 patients included in our study only $60 \%$ had positive SARS-CoV-2 PCR test at admission. Low sensitivity of PCR test for SARS-CoV-2 infection detection was previously described in multiple studies [11,12].

Chest CT is considered a gold standard diagnostic investigation for COVID-19-associated pneumonia [13-16]. However, this type of imaging has limited availability, requires patient transportation and can produce long-term effects due to radiation exposure. Bedsides, LUS for COVID-19 diagnosis has some important benefits. It is readily available, portable machines can be used and it is not expensive. Common features of pulmonary involvement that are detectable on LUS include pleural thickening with subpleural consolidations, patches of single or confluent B-lines, and consolidations that are especially prominent in the lower lobes. Preliminary results of some studies indicate a strong correlation between LUS results and chest CT findings. [17-21]. The results of our study also show a good correlation between the LUS and chest CT scores. Yale Tung-Chen et al. also described a strong correlation of LUS and CT scores in 51 patients with COVID-19 (ICC, 0.803 ; 95\% CI: $0.60-0.90 ; \mathrm{p}<0.001)$, which is similar to our findings $[5,9,22]$.

At this time there is no single established algorithm for performing LUS to evaluate lung involvement in patients with COVID-19 disease. Several approaches have been proposed, including scanning 12, 14 or 16-lung zones. In our study we used 16-lung zones scanning protocol as it has been shown to be highly effective and informative in the earlier studies of patients with acute respiratory distress syndrome (ARDS) [10,18,23].

LUS is operator-dependent and should be performed and analyzed by a highly qualified professional. Furthermore, LUS mostly evaluates pleural changes and deeper lesions could be missed since ultrasound waves cannot penetrate highly aerated lungs. Ultrasound findings also depend on clinical characteristics of the patient; obesity and subcutaneous emphysema can interfere with passage of US waves and make the assessment more difficult.

\section{Conclusion}

LUS is a useful tool to detect and monitor lung involvement in patients with COVID-19 related lung disease. However, currently there are only limited data regarding its precision as a diagnostic test and its overall clinical significance. Due to its ready availability, low cost and avoidance of ionizing radiation, LUS has value in the rapid assessment of severely ill patients in the ICU and identifying early signs of COVID-19 related pulmonary involvement, which is very important for triaging patients in the emergency department. Despite these reports and the widespread use of LUS in patients with COVID-19 related lung involvement, it is important to keep in mind the potential drawbacks of this method and correlate LUS data with clinical findings. As such, in order to standardize and refine the use of LUS in patients with coronavirus infection further studies and analyses are needed.

\author{
Abbreviations \\ ALT: alanine aminotransferase; \\ AST: aspartate aminotransferase; \\ AUC: area under the ROC-curve; \\ BMI: body mass index; \\ CAD: coronary artery disease; \\ COPD: chronic obstructive pulmonary disease; \\ COVID-19: corona virus disease 2019; \\ CT: computed tomography; \\ eGFR: estimated glomerular filtration rate; \\ ICC: intraclass correlation coefficient; \\ ICU: intensive care unit; \\ INR: international normalized ratio; \\ IQR: interquartile range; \\ LUS: lung ultrasound; \\ PT: prothrombin time; \\ PTT: partial thromboplastin time; \\ RNA: ribonucleic acid; \\ ROC: receiver operating characteristic; \\ RT-PCR: reverse transcription polymerase chain reaction; \\ SARS-CoV-2: severe acute respiratory syndrome coronavirus 2; \\ WBC: white blood cell.
}

\section{References}

1. Volpicelli G, Gargani L. Sonographic signs and patterns of COVID-19 pneumonia. Ultrasound J 2020;12:22.

2. Volpicelli G, Lamorte A, Villén T. What's new in lung ultrasound during the COVID-19 pandemic. Intensive Care Med 2020;46:1445-8.

3. Sultan LR, Sehgal CM. A Review of early experience in lung ultrasound in the diagnosis and management of COVID-19. Ultrasound Med Biol 2020;46:2530-45.

4. World Health Organization. Clinical management of severe acute respiratory infection (SARI) when COVID-19 disease is suspected: interim guidance, 13 March 2020. Accessed: March 13, 2020. Available from: https://apps.who.int/iris/ handle $/ 10665 / 331446$

5. Nouvenne A, Zani MD, Milanese G, Parise A, Baciarello M, Bignami EG, et al. Lung ultrasound in COVID-19 pneumonia: Correlations with chest $\mathrm{CT}$ on hospital admission. Respiration 2020;99:617-24.

6. Zhang R, Ouyang H, Fu L, Wang S, Han J, Huang K, et al. CT features of SARS-CoV-2 pneumonia according to clinical presentation: a retrospective analysis of 120 consecutive patients from Wuhan city. Eur Radiol 2020;30:4417-26.

7. Li K, Fang Y, Li W, Pan C, Qin P, Zhong Y, et al. CT image visual quantitative evaluation and clinical classification of coronavirus disease (COVID-19). Eur Radiol 2020;30:440716.

8. Bao C, Liu X, Zhang H, Li Y, Liu J. Coronavirus disease 2019 (COVID-19) CT findings: A systematic review and metaanalysis. J Am Coll Radiol 2020;17:701-9.

9. Tung-Chen Y, Martí de Gracia M, Díez-Tascón A, AlonsoGonzález R, Agudo-Fernández S, Parra-Gordo ML, et al. Correlation between chest computed tomography and lung ultrasonography in patients with coronavirus disease 2019 (COVID-19). Ultrasound Med Biol 2020;46:2918-26.

10. Bouhemad B, Mongodi S, Via G, Rouquette I. Ultrasound for 'lung monitoring' of ventilated patients. Anesthesiology 2015; 122:437-47.

11. Long C, Xu H, Shen Q, Zhang X, Fan B, Wang C, et al. Diagnosis of the coronavirus disease (COVID-19): rRT-PCR 
or CT? Eur J Radiol 2020;126:108961.

12. Ai T, Yang Z, Hou H, Zhan C, Chen C, Lv W, et al. Correlation of chest CT and RT-PCR testing in coronavirus disease 2019 (COVID-19) in China: a report of 1014 cases. Radiology 2020;296:E32-40.

13. Chung M, Bernheim A, Mei X, Zhang N, Huang M, Zeng X, et al. CT Imaging features of 2019 novel coronavirus (2019nCoV). Radiology 2020;295:202-7.

14. Bernheim A, Mei X, Huang M, Yang Y, Fayad ZA, Zhang N, et al. Chest CT findings in coronavirus disease-19 (COVID19): relationship to duration of infection. Radiology 2020;295:200463.

15. Colombi D, Bodini FC, Petrini M, Maffi G, Morelli N, Milanese $\mathrm{G}$, et al. Well-aerated lung on admitting chest CT to predict adverse outcome in COVID-19 pneumonia. Radiology 2020;296:E86-96.

16. Rubin GD, Ryerson CJ, Haramati LB, Sverzellati N, Kanne JP, Raoof $\mathrm{S}$, et al. The role of chest imaging in patient management during the COVID-19 pandemic: A multinational consensus statement from the Fleischner Society. Chest 2020;158:106-16.

17. Allinovi M, Parise A, Giacalone M, Amerio A, Delsante M, Odone A, et al. Lung ultrasound may support diagnosis and monitoring of COVID-19 pneumonia. Ultrasound Med Biol 2020;46:2908-17.

18. Gargani L, Soliman-Aboumarie H, Volpicelli G, Corradi F,
Pastore MC, Cameli M. Why, when, and how to use lung ultrasound during the COVID-19 pandemic: enthusiasm and caution. Eur Hear J Cardiovasc Imaging 2020;21:941-8.

19. Piscaglia F, Stefanini F, Cantisani V, Sidhu PS, Barr R, Berzigotti A, et al. Benefits, open questions and challenges of the use of ultrasound in the COVID-19 pandemic era. The views of a panel of worldwide international experts. Ultraschall Med 2020;41:228-36.

20. Soldati G, Smargiassi A, Inchingolo R, Buonsenso D, Perrone $\mathrm{T}$, Briganti DF, et al. Proposal for international standardization of the use of lung ultrasound for COVID-19 patients; a simple, quantitative, reproducible method. J Ultrasound Med 2020;39:1413-9.

21. Poggiali E, Dacrema A, Bastoni D, Tinelli V, Demichele E, Mateo Ramos P, et al. Can lung US help critical care clinicians in the early diagnosis of novel coronavirus (COVID-19) pneumonia? Radiology 2020;295:E6.

22. Lieveld AWE, Kok B, Schuit FH, Azijli K, Heijmans J, van Laarhoven A, et al. Diagnosing COVID-19 pneumonia in a pandemic setting: Lung ultrasound versus CT (LUVCT) - a multicentre, prospective, observational study. ERJ Open Res 2020;6:00539-2020.

23. McDermott C, Daly J, Carley S. Combatting COVID-19: is ultrasound an important piece in the diagnostic puzzle? Emerg Med J 2020;37:644-9. 